\title{
Activated regulatory $T$ cells suppress effector NK cell responses by an IL-2-mediated mechanism during an acute retroviral infection
}

\author{
Elisabeth Littwitz-Salomon, Ilseyar Akhmetzyanova, Cecilia Vallet, Sandra Francois, Ulf Dittmer \\ and Kathrin Gibbert ${ }^{*}$
}

\begin{abstract}
Background: It is well established that effector T cell responses are crucial for the control of most virus infections, but they are often tightly controlled by regulatory T cells (Treg) to minimize immunopathology. NK cells also contribute to virus control but it is not known if their antiviral effect is influenced by virus-induced Tregs as well. We therefore analyzed whether antiretroviral NK cell functions are inhibited by Tregs during an acute Friend retrovirus infection of mice.

Results: Selective depletion of Tregs by using the transgenic DEREG mouse model resulted in improved NK cell proliferation, maturation and effector cell differentiation. Suppression of NK cell functions depended on IL-2 consumption by Tregs, which could be overcome by specific NK cell stimulation with an IL-2/anti-IL-2 mAb complex.

Conclusions: The current study demonstrates that virus-induced Tregs indeed inhibit antiviral NK cell responses and describes a targeted immunotherapy that can abrogate the suppression of NK cells by Tregs.
\end{abstract}

Keywords: NK cells, Friend retrovirus, Regulatory T cells, IL-2 consumption, Treg suppression

\section{Background}

Natural killer (NK) cells are innate effector lymphocytes that survey the body and destroy viral pathogens without previous sensitization. Activation of NK cells is mediated by a repertoire of competing activating and inhibitory signals of germline-encoded receptors. This dynamic balance between inhibitory and activating receptors allows NK cells to recognize and eliminate infected or tumor cells while leaving out normal unaltered cells. Activated NK cells produce proinflammatory cytokines such as interferon- $\gamma$ (IFN- $\gamma)$ or tumor necrosis factor- $\alpha$. They can also induce apoptosis in target cells by the expression of cytotoxic molecules like granzymes (Gzm) or perforin or death receptor ligands on their cell surface including tumor necrosis factor related apoptosis inducing ligand (TRAIL) and Fas ligand. Potent activation and proliferation of NK cells depends on Interleukin (IL)-2,

*Correspondence: Kathrin.gibbert@uni-due.de

Institute of Virology of the University Hospital in Essen, University of Duisburg-Essen, Essen, Germany originally described as a T cell growth factor $[1,2]$. IL-2 influences different lymphocyte subsets during immune responses, differentiation, survival and homeostasis. The IL-2 receptor is comprised of three subunits-alpha (CD25) [3], beta (CD122) and gamma (CD132) [4-6]. CD122 is broadly expressed on NK cells whereas CD25 is highly expressed on activated $\mathrm{T}$ cells, especially on a distinct $\mathrm{CD} 4^{+} \mathrm{T}$ cell population called regulatory $\mathrm{T}$ cells (Tregs) [4-6]. Tregs are crucial for maintaining peripheral tolerance, preventing autoimmune diseases and limiting chronic inflammation $[7,8]$. However, by inhibition of virus-specific effector $\mathrm{T}$ cells the suppressive activity of Tregs can also be associated with progressive $T$ cell exhaustion and establishment of chronic viral infections, as shown for human immunodeficiency virus (HIV) or hepatitis $\mathrm{C}$ virus infection of humans or the murine Friend retroviral (FV) infection [9-12]. Interestingly, Tregs do not merely suppress $\mathrm{T}$ cells but can also inhibit NK cell functions in autoimmune diseases, cancer and pregnancy $[13,14]$. Particularly with regard to viral infections, NK cells contribute to the control of HIV-1 
through recognition of infected cells by activating and inhibitory killer immunoglobulin-like receptors (KIRs) [15-18]. The important role of NK cells in controlling retroviral infections was demonstrated in studies showing that polymorphisms in KIRs as well as active suppression of ligand expression for the activating natural killer group 2 member D (NKG2D) receptor by HIV-1 resulted in reduced NK cell activity in HIV-1 infection $[19,20]$. In our previous work, we also demonstrated an antiretroviral function of murine NK cells during the early phase of infection with FV [21].

It was recently described that Tregs control a subpopulation of $\mathrm{CD}_{127}{ }^{+} \mathrm{NK}$ cells in naive mice [22]. Another study also showed Treg-mediated NK cell suppression during homeostasis, which was not seen during murine cytomegalovirus (CMV) infection [23]. However, to define the influence of Tregs on NK cell responses in viral infections, Tregs have to be experimentally depleted during an ongoing infection that induces potent Treg responses. We used the well-established FV mouse model, in which a strong activation and expansion of non-virus-specific natural Tregs occurs during the acute FV infection [24]. The FV complex consists of a replication-competent but apathogenic Friend murine leukemia virus and a pathogenic but replication-defective spleen focus-forming virus. Resistant mice, such as C57BL/6, are able to recover from acute infection, but develop a persistent infection [25]. During the acute phase of FV infection, antiviral $\mathrm{CD} 8^{+} \mathrm{T}$ cells are required for the control of viral replication. However, activated Tregs subsequently start to suppress the effector functions of $\mathrm{CD}^{+}{ }^{-} \mathrm{T}$ cells, which contribute to the establishment of viral chronicity [9, 26-29].

Here, we investigated the suppressive capacity of virus-induced Tregs on NK cell responses during acute FV infection taking advantage of the transgenic DEREG mouse model in which Tregs can be selectively depleted by diphtheria toxin injection [30]. We identified a strong increase of NK cell maturation, activation and effector functions in Treg-depleted mice. Suppression by Tregs, which was mediated by IL- 2 consumption, led to increased viral loads and reduced cytotoxicity of NK cells in vivo. This suppression could be overcome by application of IL-2/anti-IL-2 mAb complex, which specifically stimulated NK cells.

Thus, defined therapies with IL-2, which specifically improve NK cell responses, should be of interest to augment NK cell responses in viral infections or cancer.

\section{Results}

NK cell frequencies inversely correlate with viral loads in Treg-depleted mice

In previous studies we showed that FV infection induced strong activation and expansion of Tregs during acute phase [12 days post infection (dpi)] of FV infection ([9]; Fig. 1a). These cells subsequently suppress $\mathrm{CD} 8^{+} \mathrm{T}$ cell responses [9]. Since no antiviral activity of NK cells could be demonstrated at the same time point [21], we determined if cytotoxic NK cells were suppressed by the expanded Tregs during acute FV infection. We infected C57BL/6 and DEREG mice with 20,000 spleen focusforming units (SFFU) of FV and subsequently depleted up to $97 \%$ of Tregs by repeated injections of diphtheria toxin (DT). As control, Tregs were also depleted in non-infected mice. In contrast to other studies [22, 23], Treg depletion during homeostasis did not significantly change proportions of NK cells in the spleen (Fig. 1a), which might be due to the different time point we performed our analysis compared to recently published studies [22, 23]. At $12 \mathrm{dpi}$, when Treg frequencies peak in the spleen [9], we analyzed proportions of NK cells in this organ. Interestingly, FV infection resulted in reduced NK cells frequencies in the spleen and no expansion of NK cells after Treg depletion during acute FV infection was observed (Fig. 1a). However, we observed an increase in NK cell frequencies in lymph nodes and peritoneum (Additional file 1c, d). We also determined the frequencies of $\mathrm{CD}_{127^{+}} \mathrm{NK}$ cells, which were previously shown to be suppressed by Tregs in naive mice [22]. We did not detect any change in the frequency of this $\mathrm{CD} 127^{+} \mathrm{NK}$ cell subpopulation after Treg ablation (data not shown). Next, we examined whether the decreased proportions of NK cells in FV-infected mice correlated with Treg frequencies or viral loads. We observed an inverse correlation of Treg frequencies and NK cell frequencies in the spleen of FV-infected mice (Fig. 1b), whereas no correlation between NK cells and viral loads was detected at 12 dpi (Fig. 1c). This suggested that virus-induced Tregs might affect NK cell frequencies during acute FV infection. To prove this, we depleted Tregs by repeating injections of DT into FV-infected DEREG mice. In contrast to Fig. 1c we found an inverse correlation between frequencies of NK cells and viral loads after Treg depletion (Fig. 1d). These results suggest that Tregs might regulate NK cell responses during acute FV infection.

\section{NK cells show enhanced activation and effector molecule expression in the absence of Tregs}

To further investigate the influence of Tregs on NK cell activation and function, we performed a phenotypic comparison of NK cells from FV-infected non-depleted and Treg-depleted mice (Fig. 2). Tregs can suppress NK cells during homeostasis [22, 23], therefore, we also used uninfected Treg-depleted and non-depleted mice as controls in the following experiments. As depicted in Fig. 2a, Additional file 2, as well as Additional file 1a and b, the proliferation of NK cells, measured by the intracellular 

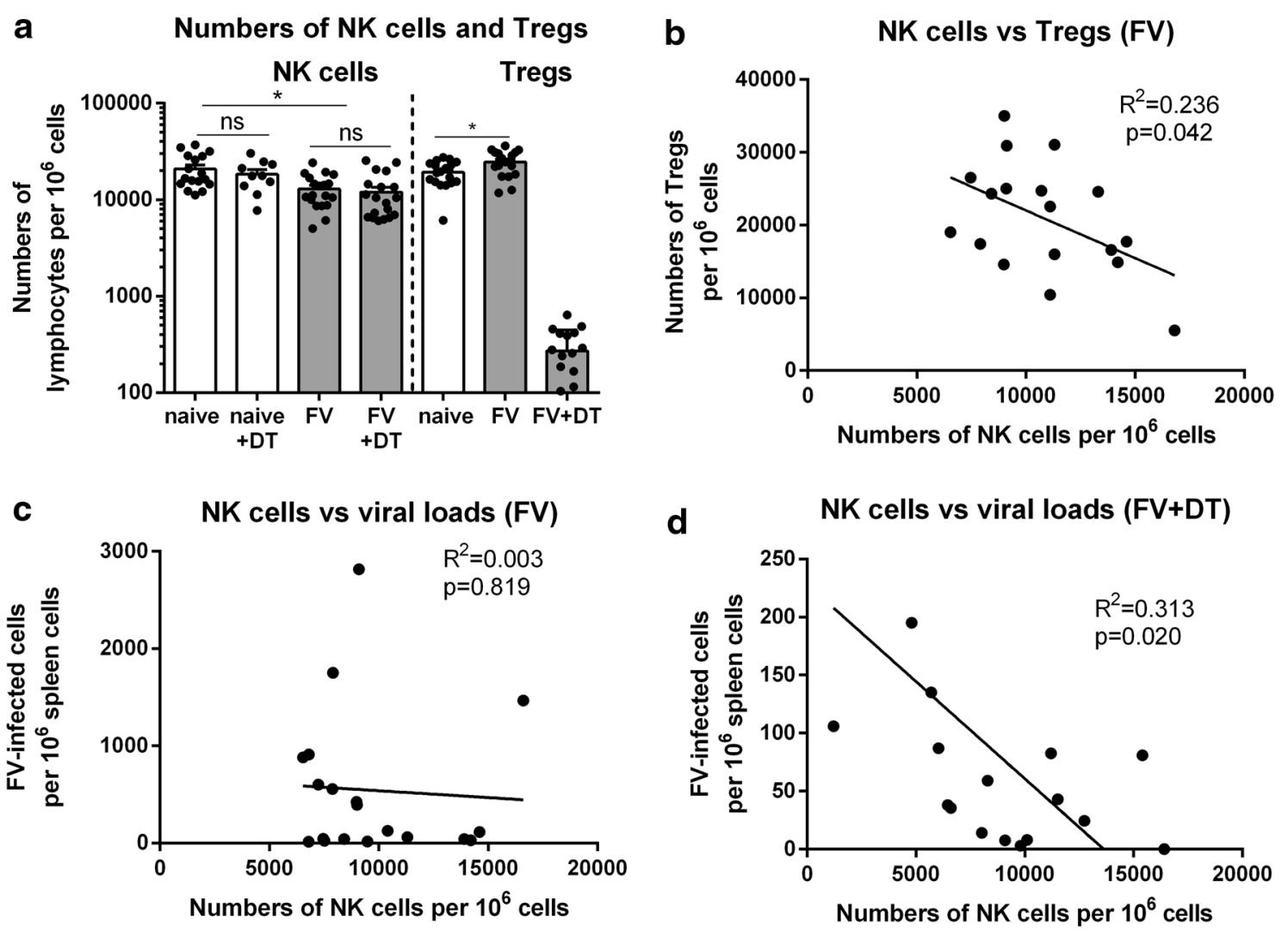

Fig. 1 Correlation of NK cell frequencies with Treg frequencies and viral loads. DEREG mice were infected with FV and depleted for Tregs by repeated injections of DT. Uninfected DEREG mice were used as controls. Splenocytes were isolated at $12 \mathrm{dpi}$ and proportions of NK cells $\left(\mathrm{CD}^{-}{ }^{-} \mathrm{CD} 49 \mathrm{~b}^{+} \mathrm{NK} 1.1^{+}\right)$and Tregs $\left(\mathrm{CD} 4^{+} \mathrm{Foxp}^{+}\right)$were determined using flow cytometry $(\mathbf{a}, \mathbf{b})$. As a control numbers of Tregs following application of DT in transgenic DEREG mice were also shown (a, right column). Individual proportions and mean ( \pm SEM) values are indicated by bars and dots. Viral loads of FV-infected mice (c) and FV-infected and Treg-ablated mice (d) were analyzed by infectious center assay and correlated to proportions of NK cells. A minimum of ten mice per group were used. Experiments were repeated at least three times. Statistically significant differences between the groups in a were determined by Kruskal-Wallis one-way analysis and Dunn's multiple comparison tests (NK cells) or by using the unpaired student's t test (Tregs) and are indicated by single asterisk for $p<0.05$ and $n$ s not significant. Statistically significant correlations were analyzed using the Pearson correlation test and results were shown in the graph.

expression of Ki-67 or the incorporation of BrdU into newly generated cells, was significantly increased in the absence of Tregs in FV-infected mice $\left(42.17 \%\right.$ Ki- $67^{+}$NK cells in Treg-depleted mice versus $27.5 \% \mathrm{Ki}-67^{+} \mathrm{NK}$ cells in non-depleted mice). We also determined if the proliferating NK cells underwent apoptosis by Annexin V staining, but we did not observe enhanced apoptosis of NK cells after Treg depletion (data not shown) indicating that newly generated NK cells $\left(\mathrm{Ki}^{6} 7^{+} \mathrm{NK}\right.$ cells; $\mathrm{BrdU}^{+}$ NK cells) post Treg-depletion do not accumulate in the spleen, but rather in lymph nodes and the peritoneum (Additional file 1c, d). Analyzing proliferation, activation, or maturation of NK cells we did not find a significant change after depletion of Tregs in uninfected mice in comparison to non-depleted mice (Fig. 2a-d). In contrast, we detected a significant increase in the expression of the early activation marker CD69 (Fig. 2b; Additional file 2) and a significant downregulation of CD62L (data not shown), demonstrating their effector phenotype in Treg-deficient mice in comparison to non-depleted control mice during acute FV infection. Comparing the same experimental groups also significant higher percentages of $\mathrm{KLRG1}^{+}$NK cells [31] (Killer cell lectin-like receptor subfamily $\mathrm{G}$ member 1 ), which represent mature NK cells, were observed in Treg-deficient mice (Fig. 2c; Additional file 2). To further characterize the differentiation state of NK cells, we analyzed the expression of the surface markers CD11b and CD27, which classify four main stages of NK cell development [32]. NK cells differentiate

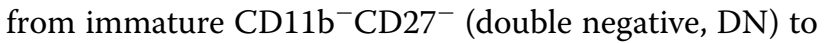
$\mathrm{CD}_{11 \mathrm{~b}}{ }^{-} \mathrm{CD} 27^{+}$through $\mathrm{CD} 11 \mathrm{~b}^{+} \mathrm{CD} 27^{+}$(double positive, $\mathrm{DP})$ to $\mathrm{CD} 11 \mathrm{~b}^{+} \mathrm{CD} 27^{-}$cells. The last two stages define 


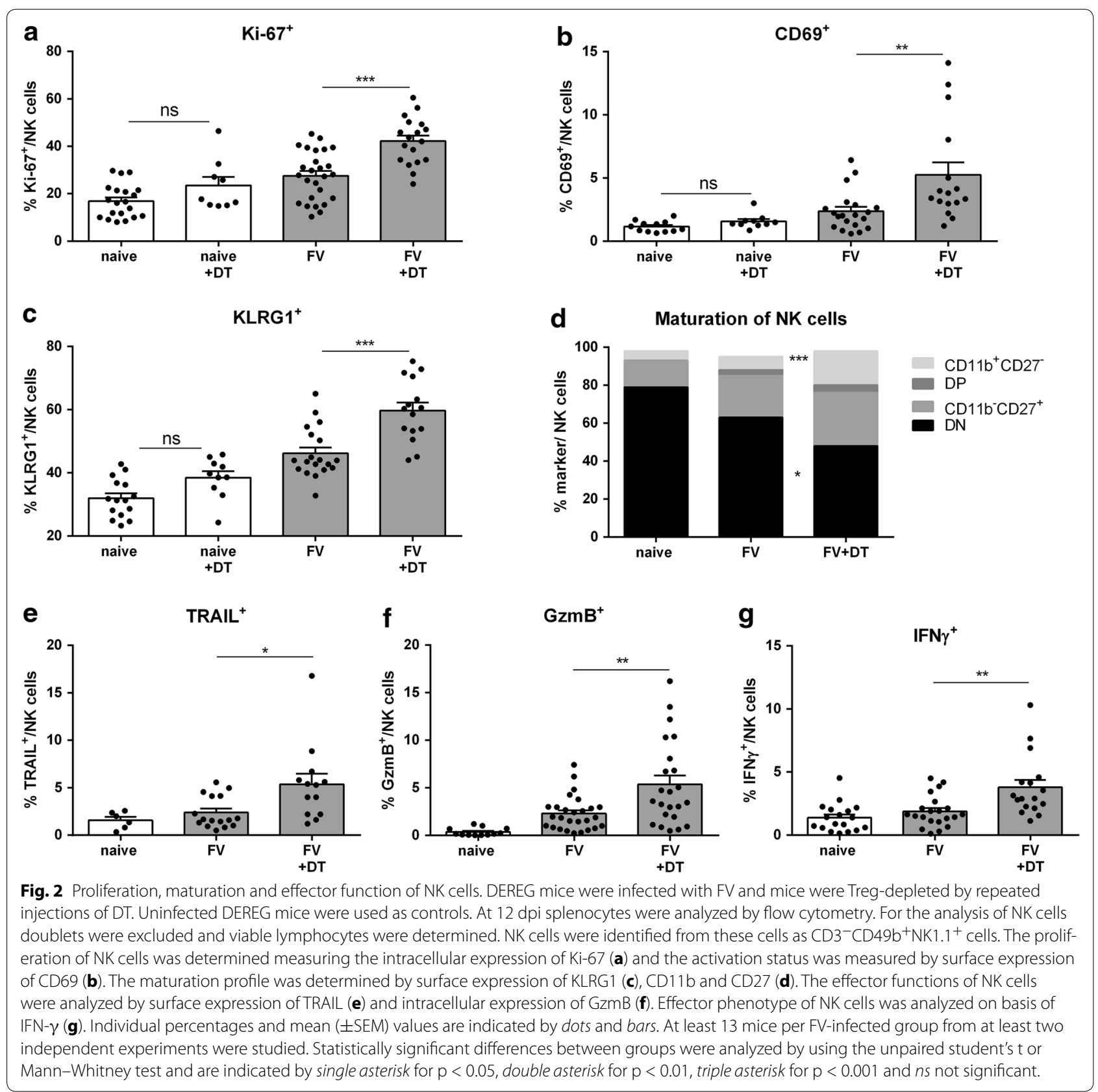

mature NK cells $\left(\mathrm{CD} 11 \mathrm{~b}^{+} \mathrm{CD} 27^{+}\right.$and $\left.\mathrm{CD} 11 \mathrm{~b}^{+} \mathrm{CD} 27^{-}\right)$ and the latter is classified as most mature or terminally differentiated NK cells [32, 33]. After Treg ablation, we observed a significant decrease in the percentage of DN immature NK cells and a significant increase in $\mathrm{CD}_{11 \mathrm{~b}}{ }^{+} \mathrm{CD} 27^{-}$terminally differentiated NK cells (18\%) compared to non-depleted FV-infected mice (7\%, Fig. 2d; Additional file 2). Similar results were obtained from an analysis of the effector phenotypes of NK cells. In the absence of Tregs, NK cells expressed significantly more TRAIL (Fig. 2e), GzmB (Fig. 2f) and IFN- $\gamma$ (Fig. 2g) in comparison to FV-infected control mice. These results demonstrate a decreased NK cell proliferation, differentiation and effector function in FV-infected mice due to a virus-induced expansion of Tregs.

Treg ablation results in potent target cell killing by NK cells To investigate whether the increased expression of effector molecules on NK cells after Treg depletion in FVinfected mice correlates with a higher killing capacity of NK cells, we performed an in vivo NK cell cytotoxicity assay. Therefore, we transferred MHC class I-deficient 
tumor cells (RMA/S cells) into the peritoneal cavity of FV-infected mice at $10 \mathrm{dpi}$, where a similar increase in NK cell effector functions, representatively shown by TRAIL expression, was observed post Treg depletion (Additional file 1e). Two days later, we re-isolated the tumor cells by peritoneal lavage and counted the remaining CFSE-labeled RMA/S. In Treg-depleted mice up to $80 \%$ of the target cells were killed (Fig. 3a; FV+DT), whereas in Treg-containing mice only $23.2 \%$ of the RMA/S tumor cells had been eliminated by NK cells (Fig. 3a, FV). To prove that this killing was NK cell dependent, NK1.1 $1^{+}$cells were depleted in addition to Tregs (FV+DT+anti-NK1.1), which strongly reduced the killing to the same background level detected in control mice (FV). These results indicate that the general cytotoxicity of NK cells was strongly enhanced after Treg ablation in FV-infected mice. To analyze if this also translates into enhanced anti-FV activity of NK cells, we performed an adoptive transfer experiment with enriched NK cells (Fig. 3b). NK cells isolated from FVinfected Treg-depleted or non-depleted mice were transferred into FV-infected wild type mice. FV-infected mice without any cell transfer were used as controls. NK cells transferred from FV-infected donors did not significantly decrease viral loads compared to controls, whereas NK cells from Treg-deficient mice mediated an 80-fold reduction in viral loads (Fig. $3 \mathrm{~b}$ ). Here, we could show that Tregs suppress antiviral NK cell cytotoxicity during an acute retrovirus infection.

\section{Tregs deprive NK cells of IL-2 during acute FV infection}

Next, we set out to analyze the mechanism of Tregmediated suppression of NK cell responses during FV infection. Previous reports by Ghiringhelli et al. [34] and Smyth et al. [35] described a TGF- $\beta$-dependent suppression of NK cells by Tregs in cancer models. Hence, we analyzed TGF- $\beta$ protein level in the serum and mRNA expression of TGF- $\beta$ and also IL-10 in the spleen during FV infection, but we could not detect any differences in the expression levels between Treg-depleted and nondepleted mice (data not shown), suggesting that TGF- $\beta$ and IL-10 do not play a role in our infection model. Then, we focused on IL-2, which influences different lymphocyte subsets, including Tregs and NK cells, in their proliferation, differentiation, and effector functions. To analyze the impact of IL- 2 on NK cell responses during acute FV infection, we depleted Tregs by repeating injections of DT and measured the IL-2 concentration in the serum of mice. Uninfected Treg-depleted and DT-treated wild type mice served as controls, but we could not measure significant increased levels of IL-2 in the serum of these mice (data not shown). However, we detected a 21-fold increase in mean IL-2 serum levels between FV-infected mice with Tregs and FV-infected mice lacking Tregs (Fig. 4a), suggesting that Tregs consume large amounts of IL- 2 or they suppress the production of IL- 2 by $C D 4^{+} \mathrm{T}$ cells [36].

IL-2 binds to the high-affinity trimeric IL-2 receptor (CD25, CD122 and CD132) and to the low affinity
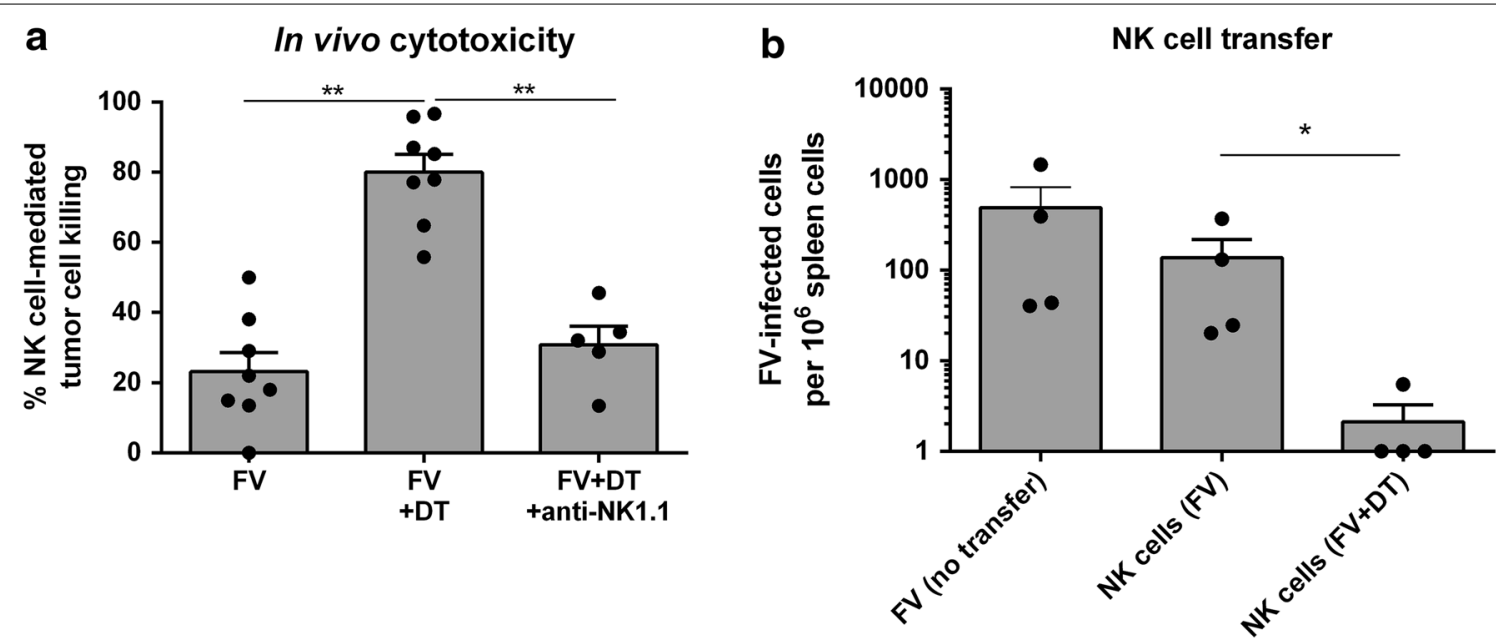

Fig. 3 NK cell dependent target cell killing. DEREG mice were infected with FV and mice were either Treg-depleted by repeated injections of DT or NK cell-depleted or Treg- and NK cell-depleted. At 10 dpi mice received $5 \times 10^{5}$ CFSE-labeled RMA/S. Two days later (12 dpi) cells were obtained by peritoneal lavage and analyzed by flow cytometry (a). At least three independent experiments were performed with at least five mice. Individual percentages and mean ( \pm SEM) values were indicated by dots and bars. b DEREG mice were infected with FV and several mice were Treg-depleted by repeated injections of DT. At 10 dpi splenic NK cells were isolated and adoptively transferred into FV-infected mice. Four days post transfer mice were sacrificed and viral loads were analyzed in the spleen. FV-infected mice, which did not receive any cell transfer, were used as control. At least four mice per group were tested. Statistically significant differences between the different transferred NK cells were analyzed via Mann-Whitney test and are indicated by single asterisk for $p<0.05$ and double asterisk for $p<0.01$. 
dimeric IL-2 receptor consisting of CD122 and CD132 [37]. Tregs express preferably the trimeric IL-2 receptor, whereas dimeric receptors are rather associated with $\mathrm{CD}^{+}{ }^{+} \mathrm{T}$ cells and NK cells. Treg depletion did not change the proportion of CD122 $2^{+} \mathrm{NK}$ cells in comparison to non-depleted mice (data not shown) during FV infection. Analysis of the IL-2R $\alpha$-chain (CD25) also revealed no changes in the expression levels after Treg depletion in uninfected and FV-infected mice (Fig. 4b, c).

\section{Specific IL-2 stimulation of NK cells mimics the effect of Treg depletion on NK cell activation and differentiation and leads to better FV control}

To confirm that IL-2 is an important factor in Treg-mediated suppression of NK cell responses, we specifically stimulated NK cells with IL-2 using a complex of antimouse IL-2 mAb (clone S4B6-1) in combination with recombinant mouse IL-2 protein. This antibody binds to the site of IL-2, which normally interacts with CD25 and thus boosts binding of IL-2 to the two other receptor subunits (CD122 and CD132) [37, 38], which are highly expressed on NK cells but not on Tregs. Application of IL-2 complex resulted in an increase in NK cell frequencies (FV+IL-2/anti-IL-2) compared to control mice (FV) (Fig. 5a), which was also seen in the expression of the proliferation marker Ki-67 (Fig. 5b). However, an additional depletion of Tregs (FV+DT+IL-2/antiIL-2) did not further change the total frequencies of NK cells in comparison to IL-2 stimulated FV-infected mice (FV+IL-2/anti-IL-2).

We also detected a significant increase in the expression of the early activation marker CD69 (Fig. 5c) and the maturation marker KLRG1 (Fig. 5d) after stimulation with the IL-2/anti-IL-2 mAb complex which were comparable to those seen in Treg-depleted mice. The combination of both treatments (FV+DT+IL-2/anti-IL-2) did not further increase the expression of both markers (Fig. 5c, d).

Next, we analyzed the differentiation state of NK cells after stimulation with IL-2 by expression of the surface molecules CD11b and CD27 (Fig. 5e). We observed a

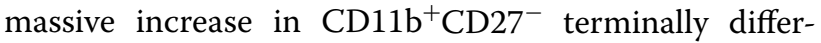
entiated NK cells (32\%) compared to control mice (7\%), which was also seen after Treg depletion $(\mathrm{FV}+\mathrm{DT})$. This effect is not further increased by additional Treg depletion (FV+DT+IL-2/anti-IL-2), suggesting that NK cells
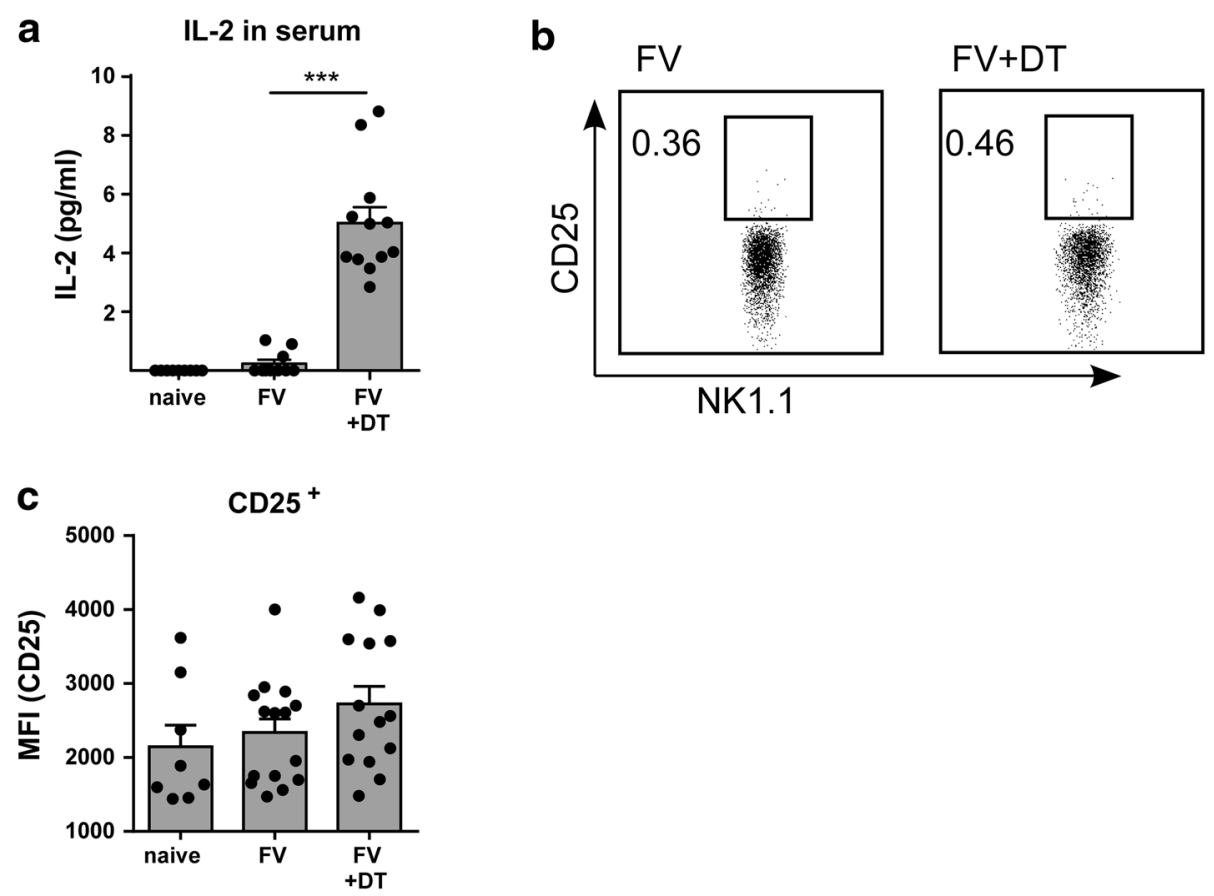

Fig. 4 Effects of Treg ablation on IL-2 and IL-2R $\alpha$-chain. DEREG mice were infected with FV and mice were Treg-depleted by repeated injections of DT. Uninfected DEREG mice were used as controls. Twelve days post infection blood and spleen were taken for analysis. Serum was analyzed for IL-2 concentration by ELISA (a). Samples of five different experiments were tested with at least ten samples per group. The increase of IL-2 concentration in serum is shown by bars and dots ( \pm SEM). Statistically significant changes were determined by Mann-Whitney test and indicated by triple asterisk for $\mathrm{p}<0.001$. Representative dot plots of CD25 expression on splenic NK cells (NK1.1 ${ }^{+} \mathrm{CD}_{9} 9 \mathrm{~b}^{+} \mathrm{CD}^{-}$) in FV-infected and FV-infected and Treg-depleted mice are shown in (b). Expression of IL-2R $\alpha$-chain (CD25) on NK cells was analyzed by flow cytometry and indicated by mean ( \pm SEM) values and dots (c). A minimum of eight mice in at least two independent experiments were analyzed. 


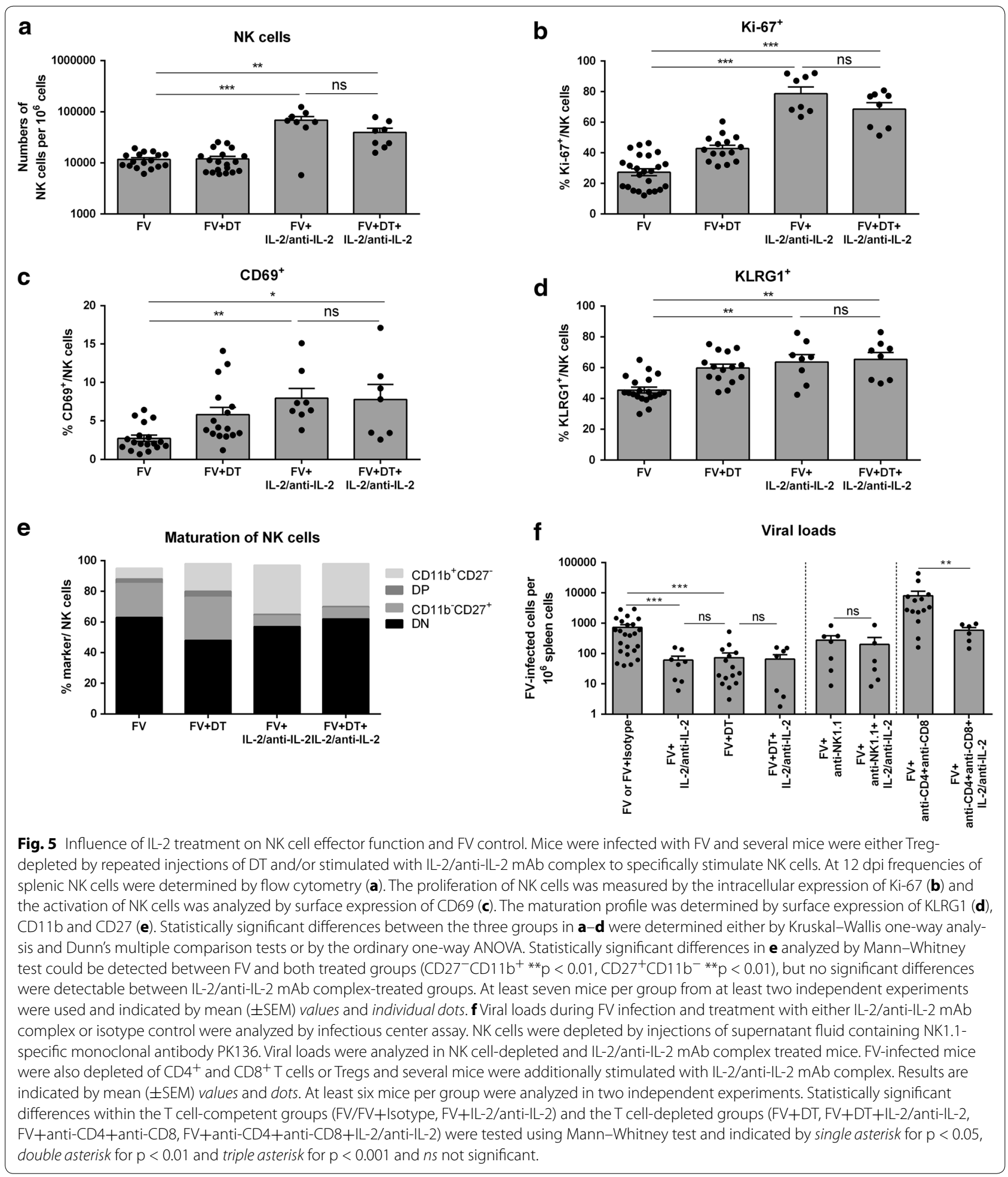

are saturated with IL-2 after application of the IL-2/antiIL-2 mAb complex. Additional depletion of Tregs might increase the level of circulating IL-2, which is not further consumed by stimulated NK cells.
Finally, we wanted to determine whether the improved NK cell activation by IL- 2 treatment has an effect on viral loads. Interestingly, specific NK cell stimulation with IL-2 in the presence of Tregs mediated a significant reduction 
in viral loads (FV+IL-2/anti-IL-2; Fig. 5f), which was comparable to the results seen in Treg-depleted mice (FV+DT; Fig. 5f). In line with our previous results of NK cell activation and effector function (Fig. 5b-e), the combination of Treg depletion and NK cell stimulation did not further decrease viral burden (FV+DT+IL-2/ anti-IL-2; Fig. 5f). To confirm that the observed reduction in the viral loads post IL-2 treatment is mediated by NK cells and not by other cells which express the IL-2 receptor, we depleted $\mathrm{CD} 8^{+}$and $\mathrm{CD} 4^{+} \mathrm{T}$ cells by injection of supernatant fluid containing CD8-specific mAb 169.4 and CD4-specific mAb YTS 191.1. We stimulated NK cells by application of IL-2/anti-IL- 2 mAb complex in these $\mathrm{T}$ cell depleted mice and determined viral loads at 12 dpi. As expected, after depletion of $\mathrm{CD} 4^{+}$ and $\mathrm{CD} 8^{+} \mathrm{T}$ cells, the viral loads significantly increased (FV+anti-CD4+anti-CD8; Fig. 5f; Additional file 3), as T cells, especially $\mathrm{CD}^{+} \mathrm{T}$ cells, are required for $\mathrm{FV}$ control at that time point [9]. An additional NK cell depletion resulted in a further rise of the viral loads (Additional file 3) demonstrating that NK cells are involved in antiviral immunity during acute FV infection. In contrast, specific NK cell stimulation with IL-2 in T cell-depleted mice (FV+anti-CD4+anti-CD8+IL-2/anti-IL-2) led to a more than 13-fold reduction in the viral loads compared to unstimulated $\mathrm{T}$ cell-depleted controls (FV+antiCD4+anti-CD8) showing that IL-2-stimulated NK cells contribute to FV control.

Additionally we injected IL-2/anti-IL-2 mAb complexes (clone JES-1) targeting Tregs to clarify that specific IL-2 stimulation of NK cells is required for FV control. In contrast to the antibody complexes used before, application of the other IL-2 antibody complex specifically activated Tregs (expression of CD43; Additional file 4c) and this did not result in reduction of viral loads (data not shown).

We also analyzed the activation status of Tregs, $\mathrm{CD} 4^{+}$ and $\mathrm{CD} 8^{+} \mathrm{T}$ cells after treatment with IL-2/anti-IL-2 mAb complexes, but we did not detect any effect of IL-2 on $\mathrm{T}$ cells compared to FV-infected control mice (Additional file $4 \mathrm{a}, \mathrm{b})$. Furthermore, NK cell depletion during IL-2/anti-IL-2 mAb complex application completely abolished IL-2-mediated antiviral immunity (Fig. 5f). This implies that the therapeutic effect of IL-2 was mediated by NK cells and could overcome the suppressive effect of virus-induced Tregs, which led to control of acute FV infection. Here, we describe a new suppressive mechanism of Tregs on NK cell functions during an acute viral infection that is dependent on IL-2 availability or IL-2 consumption by Tregs.

\section{Discussion}

Efficient immune protection against pathogens such as viruses is usually provided by a successful interaction of innate and adaptive immunity. NK cells participate in the rapid control of early viral replication, whereas virus-specific $T$ cells undergo clonal expansion and thus contribute later to antiviral immunity. A potent immune response has to be counter-regulated to prevent immunopathology. This immune control is efficiently mediated by Tregs, which dampen pathogen-specific effector $\mathrm{T}$ cell responses. Besides preventing immunopathology, Treg activity can also contribute to inefficient clearance of pathogens and the establishment of chronic viral infections [39]. However, whether Tregs also influence the initial antiviral response of NK cells was not known.

The current study shows for the first time that NK cell responses are suppressed by virus-induced Tregs during an acute retroviral infection. Specific depletion of Tregs during acute FV infection resulted in improved NK cell maturation, differentiation and effector functions (Fig. 2). The suppression of NK cells was mediated by Treg-driven IL-2 consumption, which can be overcome by specific stimulation of NK cells with an IL-2/anti-IL-2 mAb complex in vivo. Others showed a Treg-mediated suppression of NK cell responses in cancer, autoimmunity or during homeostasis, whereas no studies on Treg-mediated NK cell suppression in viral infections existed. Only one recent study using CMV-infected mice analyzed the impact of Treg depletion on NK cell responses. Lindenberg and colleagues [23] showed that during acute mouse CMV infection Tregs suppress only $\mathrm{T}$ cell responses, whereas NK cell responses were not affected by Tregs. However, CMV infection of mice is not a very useful model to study the interaction of Tregs and NK cells because the virus does not efficiently activate or expand Tregs [40]. The effect of virus-induced Tregs on anti-viral NK cell responses can only be investigated in an infection model with strong Treg responses, like the FV mouse model $[9,41]$. In a mouse cancer model, $\mathrm{CD} 4{ }^{+} \mathrm{CD} 25^{+}$ Tregs inhibited NKG2D-mediated NK cell cytotoxicity by TGF- $\beta$ release and subsequent tumor rejection and metastasis inhibition [35]. Similar results were also shown by Ghiringhelli and colleagues [34] who described suppression of NK cell cytotoxicity by Tregs in a mouse B16 tumor model and human melanoma patients. Our current data and the described studies imply that negative regulation of NK cell responses by Tregs might be a common feature in infections and cancer. Besides cytotoxic $\mathrm{T}$ cells, also NK cell cytotoxicity is controlled by Tregs most likely to avoid immunopathology.

Several molecular mechanisms of Treg suppression of NK cells have been discussed. One mechanism used by Tregs to suppress antitumor responses depends on granzyme $\mathrm{B}$ and perforin and thus on direct killing of NK cells and $\mathrm{CD}^{+} \mathrm{T}$ cells by Tregs in allogenic B16 and RMA/S tumors [42]. However, in FV-infected mice, 
no expression of granzymes has been found in activated Tregs [43], suggesting that other mechanisms of suppression play a more important role. As TGF- $\beta$ is an important factor in Treg-mediated suppression of NK cells in cancer models $[34,35]$, we also analyzed the expression of TGF- $\beta$ in FV-infected and additionally Treg-depleted animals. We did not detect any differences after depletion of Tregs (data not shown), indicating that TGF- $\beta$ is not involved in the suppression of antiviral NK cell effector functions during FV infection.

In the mouse B16 melanoma model described above, the authors also demonstrated that an in vitro stimulation of PBMC cultures with IL-2 neutralized the Treg-mediated suppression of NK cells suggesting an important role of IL-2 in Treg-NK cell interaction [34]. Our findings and recent studies suggest a cellular interplay of Tregs, $\mathrm{CD} 4^{+}$ helper T cells and NK cells. Tregs do not produce IL-2 by themselves, but they consume IL-2 which is mainly produced by $\mathrm{CD} 4^{+} \mathrm{T}$ cells. The hypothesis is that Tregs dampen the bioavailability of IL-2 by massive consumption of the cytokine or inhibition of IL-2 production by $\mathrm{CD} 4^{+} \mathrm{T}$ cells and thus indirectly inhibit NK cells due to IL-2 deprivation. There is evidence from a mouse diabetes model showing the importance of Treg suppression and IL-2 consumption in an autoimmune disease [44]. Here, Tregs limited the availability of IL- 2 in the pancreatic islets, which dampened activation and effector functions of infiltrating NK cells. However, in the absence of Tregs, IL- 2 produced by infiltrating $\mathrm{CD}^{+}$helper $\mathrm{T}$ cells activated NK cells and enhanced their IFN- $\gamma$ production which leads to the onset of diabetes. This effect was prevented by application of an IL-2 antagonist in Tregdepleted mice demonstrating the importance of IL-2 in regulating NK cell functions. Others found that depletion of Tregs in Foxp3.DTR mice did not change NK cell tolerance to self- or non-self-ligands, whereas missingself-induced cytotoxicity of NK cells was enhanced in Treg-depleted mice. They showed that this was dependent on $\mathrm{CD}^{+}$helper T cells producing IL- 2 and that regulating IL-2 levels may be a mechanism of Tregs to tune NK cell activity [45]. During acute FV infection, IL-2 is mainly produced by $\mathrm{CD} 4^{+}$helper $\mathrm{T}$ cells $[24,36]$, which was subsequently consumed by expanded Tregs. NK cells only received sufficient IL-2 signaling for full activation when the IL-2 consumption by Treg was limited. Thus, our previous data and the current results suggest an interplay of $\mathrm{CD}^{+}$helper $\mathrm{T}$ cells, Tregs and NK cells also in viral infections with IL-2 being the specific link between these cell populations. Gasteiger et al. [22] described that Tregs mainly control a very specific NK cell subpopulation $\left(\mathrm{CD} 127^{+}\right)$during homeostasis, which was also dependent on the availability of IL-2. However, this population of NK cells does not seem to play a major role during FV infection, as it accounted for only $0.5 \%$ of all NK cells in infected mice, and this frequency did not change after depletion of virus-induced Tregs.

Immunotherapy with IL-2 is already used in the clinics to treat patients with metastatic melanoma and renal cell carcinoma with objective clinical response rates of $15-25 \%$. As IL-2 has multiple activities on various lymphocytes, treatment with IL-2 results in a variety of different side effects. Many trials have been performed to reduce these by changing the dose, route, or chemical modification of IL-2 or adding additional immunomodulators; however, they were not successful in enhancing the clinical responses [46]. Another attempt is to selectively target distinct cell populations by IL-2 immunotherapy. The IL-2 receptor consists of three subunits: CD25 $(\alpha)$, CD122 ( $\beta)$ and CD132 $(\gamma)$ [38]. CD25 is highly expressed on Tregs, but hardly expressed on NK cells and memory-phenotype $\mathrm{CD}^{+} \mathrm{T}$ cells [47]. Addition of anti-IL-2 mAb to recombinant IL-2 can target the cytokine to specific cell subsets. In mice, two different clones of anti-IL-2 mAb are available that bind to different sites of the cytokine. Clone JES6-1 shields the CD122 binding site which further targets this antibody to $\mathrm{CD} 25$, whereas clone S4B6 interferes with the CD25 binding site of IL-2 and thus leads to preferential CD122 binding [38, 47]. Application of one of these antibodies together with recombinant IL-2 can therefore target IL-2 to Tregs (clone JES6-1) or to NK cells or memoryphenotype $\mathrm{CD}^{+} \mathrm{T}$ cells (clone S4B6). In our study, we used clone S4B6 to specifically stimulate NK cells and reduce binding to Tregs. We observed a significant activation, maturation and differentiation of NK cells upon this IL-2 immunotherapy (Fig. 5), whereas no stimulatory effect was detected on different $T$ cell subsets (Additional file 2). The selective IL-2 stimulation of NK cells reduced viral loads (Fig. 5f) and could overcome suppressive Treg activity suggesting that this might be an interesting new approach for the treatment of infectious diseases. Studies in a B16 melanoma model have shown that immunotherapy with an IL-2/anti-IL-2 mAb complex only binding to the IL-2 $\beta \gamma$ receptor abrogated IL-2 therapy mediated side effects (pulmonary edema) and led to a vigorous activation of cytotoxic immune cells with strong antitumor reactivity [5]. In their mouse model for autologous hematopoietic stem cell transplantation, Newman and colleagues demonstrated that vaccination with lymphoma cells secreting antibodies in combination with IL-2/anti-IL-2 mAb complexes (clone S4B6) induced strong antitumor responses of cytotoxic cells $\left(\mathrm{CD}^{+}\right.$ $\mathrm{T}$ cells and NK cells) and thus prevented the establishment of hematologic cancers [48]. Others compared the efficacy of the IL-2/anti-IL-2 mAb complex with IL-2 treatment alone in a B16 melanoma mouse model. The 
combined treatment could markedly enhance cytotoxicity of $\mathrm{CD} 8^{+} \mathrm{T}$ cells and partially protect mice from tumor metastasis compared to IL-2 treatment alone [49]. Application of IL-2/anti-IL-2 mAb (clone S4B6) complexes strongly extends the half-life of soluble IL-2 and prevents binding to CD25 on Tregs resulting in a potent improvement of the biological activity of the complex compared to soluble IL-2 [47]. However, these studies did not discuss the role of Tregs in their infection models [50].

Similar antibodies for IL-2 to target different cell subsets also exist for the use in humans. Complexes of human IL-2 and anti-IL-2-mAB clone MAB602 closely resemble S4B6-IL-2 complexes targeting IL-2 to CD122 on the surface of immune cells. An equivalent for JES6-1 directing IL-2 to CD25 (mAb 5344) does also exist. Also, two mutated isoforms of human IL-2 were developed with increased binding to either CD25 or CD122 [51, 52] implying that targeted IL-2 immunotherapies in humans may be feasible in the future.

To our knowledge, this is the first study showing that immunotherapy with an IL-2/anti-IL-2 mAb complex can overcome Treg suppression and improve NK cell responses in an acute retroviral infection. The specific stimulation of NK cells by IL-2 can result in improved control of viral infections. Such a therapeutic approach has a clear advantage over Treg depletion or manipulation during viral infections, as it may significantly reduce the risk of inducing immunopathologies. Using antibodycytokine-complexes to specifically target distinct immune cell populations may have a strong impact in future therapies against infections and cancer, as side-effects in patients can be reduced and clinical responses improved.

\section{Conclusion}

Natural killer (NK) cells, which belong to the innate arm of the immune system, can contribute to the control of many viral infections. NK cells can directly kill virus-infected cells and thus reduce viral replication. However, in infections with retroviruses, like HIV, NK cells are not sufficient to prevent pathology. Here, we showed that NK cell effector functions are strongly inhibited by virus-induced regulatory $\mathrm{T}$ cells leading to failure of NK cells to control retroviral infection. We described IL-2-mediated suppression mechanism by regulatory $\mathrm{T}$ cells which can be overcome by NK cell-specific stimulation with IL-2 complexes. This new therapeutic approach to target specific immune cells by antibody-cytokine-complexes might have great potential for the treatment of many viral infections or cancer.

\section{Methods}

\section{Ethics statement}

Animal experiments were performed in strict accordance with the German regulations of the Society for
Laboratory Animal Science (GV-SOLAS) and the European Health Law of the Federation of Laboratory Animal Science Associations (FELASA). The protocol was approved by the North Rhine-Westphalia State Agency for Nature, Environment and Consumer Protection (LANUF) (Permit Number: G1341/12). All efforts were made to minimize suffering.

\section{Mice and virus}

Experiments were done using sex- and age-matched inbred C57BL/6 (B6, Harlan Laboratories, Germany) and wild type and transgenic DEREG mice [30]. At the beginning of the experiments, mice were $6-10$ weeks old. The FV stock used in these experiments was a FV complex containing B-tropic Friend murine leukemia helper virus and polycythemia-inducing spleen focus-forming virus. The stock was prepared as a $15 \%$ spleen cell homogenate from $\mathrm{BALB} / \mathrm{c}$ mice infected 14 days previously with 3,000 SFFU of FV. Mice were injected intravenously with $0.15 \mathrm{ml}$ phosphate-buffered saline (PBS) containing 20,000 SFFU of FV. The virus stock did not contain lactate dehydrogenase-elevating virus. Mice were sacrificed at $12 \mathrm{dpi}$ by cervical dislocation.

\section{Detection of FV-infected cells in spleen}

Infectious centers (IC) were detected by tenfold dilutions of single-cell suspensions onto Mus dunnis cells. Cultures were incubated for 3 days, fixed with ethanol, stained with F-MuLV envelope-specific monoclonal antibody 720 and developed with peroxidase-conjugated goat antimouse antibody and aminoethylcarbazol to detect foci [53].

\section{IL-2 concentration in the serum}

For detection of IL-2 concentration in FV-infected mice, sera were harvested at 12 dpi. Cytokine concentration was measured by an IL-2 ELISA (eBioscience) according to the manufacturer's protocol.

\section{Flow cytometry staining}

Cell surface staining was performed using the following antibodies: anti-CD3 (17A2, eBioscience), anti-CD4 (GK1.5, eBioscience), anti-CD8 (53-6.7, eBioscience), anti-CD11b (M1/70, BD Bioscience), anti-CD25 (PC61, BD Bioscience), anti-CD27 (LG.3A10, BioLegend), antiCD43 (1B11, BioLegend), anti-CD49b (DX5, eBioscience), anti-CD69 (H1.2F3, eBioscience), anti-CD127 (AFR34, eBioscience), anti-KLRG1 (2F1, eBioscience), anti-NK1.1 (PK136, BD Bioscience), anti-TRAIL (N2B2, eBioscience). Dead cells were excluded from analysis via fixable viability dye (eBioscience). For intracellular IFN- $\gamma$ staining, cells were stimulated with Ionomycin (500 ng/ $\mathrm{ml})$, PMA (25 $\mathrm{ng} / \mathrm{ml})$ and Brefeldin A $(2 \mu \mathrm{g} / \mathrm{ml})$ and 
incubated for $3 \mathrm{~h}$ at $37^{\circ} \mathrm{C}$. For intracellular staining of IFN- $\gamma$ (XMG1.2, eBioscience) and GzmB (clone GB11, Life technologies), cells were fixed and permeabilized with CytoFix/CytoPerm (BD Bioscience) for $10 \mathrm{~min}$. Intranuclear fixation was performed following the manufacturer's instruction (Foxp3/Transcription Factor Fixation/Permeabilization, eBioscience). Tregs were stained with antibodies against Foxp3 (FJK-16S, eBioscience) and proliferation was detected using Ki-67 (SolA15, eBioscience). Data were acquired on LSR II flow cytometer (BD Bioscience) and analyses were performed using FACSDiva (BD Bioscience) and Flow Jo (Tree Star, USA) software.

\section{BrdU treatment and staining}

5-Bromo-2'-deoxyuridine (BrdU, Sigma) was added to the drinking water of mice and was replaced every day $(40 \mathrm{mg})$. In addition, mice were injected with BrdU every day starting at day 9 (i.p., $1 \mathrm{mg}$ ). BrdU staining was performed with BrdU Flow Kit (BD Pharmingen) according to the manufacturer's protocol. Staining of cells was done with anti-BrdU (Clone BU20A, eBioscience) and acquired on LSR II flow cytometer (BD Bioscience).

\section{IL-2 receptor stimulation}

Specific stimulation of NK cells were obtained by intraperitoneal inoculation of $50 \mu \mathrm{g}$ anti-mouse IL-2 monoclonal antibody (Clone S4B6-1 or JES6-1, BioXCell) and $1 \mu \mathrm{g}$ of recombinant mouse IL-2 (carrier-free, eBioscience) in $500 \mu \mathrm{l}$ of PBS. Control groups received $50 \mu \mathrm{g}$ of isotype control (rat IgG2a, BioXCell). Mice were injected every other day starting from day 7 to day 11 post infection.

\section{NK cell transfer}

Splenic NK cells from FV-infected mice were enriched using NK cell isolation Kit II (Miltenyi Biotec) according to the manufacturer's protocol. Intravenous injection of $2 \times 10^{6} \mathrm{NK}$ cells was performed at $10 \mathrm{dpi}$ and viral loads were analyzed 4 days later. Transferred cells contained more than $85 \%$ of NK cells and only $2 \%$ of T cells.

\section{Depletion of T cells}

For depletion of $\mathrm{CD}^{+} \mathrm{T}$ cells mice were injected intraperitoneally with $0.35 \mathrm{ml}$ supernatant fluid containing CD8-specific mAb 169.4 every other day. Intraperitoneal injections of $0.15 \mathrm{ml}$ CD4-specific mAb YTS 191.1 specifically deplete the $\mathrm{CD} 4^{+} \mathrm{T}$ cell population. At the days of analysis more than $98 \%$ of $\mathrm{CD}^{+} \mathrm{T}$ cells and at least $90 \%$ of $\mathrm{CD}^{+} \mathrm{T}$ cells were depleted in the spleen. To deplete regulatory $\mathrm{T}$ cells transgenic DEREG mice were injected intraperitoneally with DT $(0.5 \mu \mathrm{g}$, Merck
Millipore) diluted in PBS every third day for three times starting at day 5 post infection. The treatment depleted over $97 \%$ of $\mathrm{CD}^{+} \mathrm{eGFP}^{+} \mathrm{T}$ cells in the spleen. Wild type DEREG mice injected with DT were used as controls.

\section{NK cell depletion}

NK cell depletion was performed as described before [21]. At the day of analysis more than $95 \%$ of NK cells $\left(\mathrm{CD}^{-} \mathrm{CD}^{-} 9 \mathrm{~b}^{+} \mathrm{NK}^{-} .1^{+}\right)$were depleted in the spleen.

\section{In vivo NK cell cytotoxicity assay}

In vivo NK cell cytotoxicity assay was performed using $5 \times 10^{5} \mathrm{RMA} / \mathrm{S}$ cells per mouse labeled with $10 \mu \mathrm{M}$ of CFSE (Vybrant ${ }^{\circledR}$ CFDA SE Cell Tracer Kit, Life technologies). RMA/S cells were injected intraperitoneally at $10 \mathrm{dpi}$. NK cell-depleted mice were used as controls. Mice were sacrificed at $12 \mathrm{dpi}$ and intraperitoneal lavage was performed with $10 \mathrm{ml}$ PBS to obtain cells. Cells were washed once, resuspended in buffer containing fixable viability dye (eBioscience) to exclude dead cells and analyzed by flow cytometry. Target cell killing was calculated as follows:

$\frac{\text { Target cells from NK cell depleted mice }- \text { Sample target cell number }}{\text { Target cells from NK cell depleted mice }} \times 100$

\section{Statistical analyses}

Statistical analyses and graphical presentations were computed with Graph Pad Prism version 6. Normal distribution was assessed using D'Agostino-Pearson omnibus test. Statistical differences between two different groups were determined by the unpaired student's $t$ test (parametric distribution) or Mann-Whitney test (nonparametric distribution). Analyses including several groups were tested using Kruskal-Wallis one-way analysis of variance on ranks and Dunn's multiple comparison test (non-parametric distribution) or the ordinary oneway ANOVA and Bonferroni's multiple comparisons test (parametric distribution). For the determination of correlating variables, linear regression and Pearson correlation were performed.

\section{Additional files}

Additional file 1: Numbers, proliferation and effector functions of NK cells during FV infection DEREG mice were infected with 20.000 SFFU of FV for 12 days. $(a, b)$ Mice were treated with 5-Bromo-2'-deoxyuridine (BrdU) starting at day 5 post FV infection. Splenocytes were isolated and NK cells (NK1.1+CD49b+CD3 - cells) stained for flow cytometry. NK cells were analyzed for BrdU+ expression $(a, b)$ and a representative graph of BrdU+ NK cell counts is shown in (a). Numbers of NK cells were analyzed in lymph nodes (c) and peritoneum (d). Representative dot plots were shown for TRAIL expression in the peritoneal lavage of FV-infected and FV-infected and Treg-depleted (DT) mice (e). Results are indicated by bars and dots +SEM. At least four mice per group were analyzed. Statistically significant differences between groups were analyzed by Mann-Whitney test and indicated by ${ }^{*}$ for $p<0.05$ and ${ }^{*}$ for $p<0.01$. 
Additional file 2: Representative dotplots of activated, matured and proliferated NK cells Splenocytes were isolated from FV-infected mice (12 dpi) and NK cells were analyzed by flow cytometry (NK1.1 +CD49b+CD3cells). NK cells were evaluated for the different, indicated markers (Ki-67, CD69, KLRG1, CD27, CD1 1b). In the first row, representative dot plots of NK cell markers are shown during FV-infected. In the middle, dot plots of NK cells from FV-infected as well as Treg-ablated mice are depicted. Fluorescence-minus-one controls (FMOs) were performed for every staining (third row).

Additional file 3: Viral loads in T cell- and NK cell-depleted mice during FV infection DEREG mice were infected with 20.000 SFFU of FV and either depleted for CD4+ and CD8+ T cells or NK cell-depleted. At 12 dpi viral loads were analyzed in spleens via infectious center assay. Individual proportions and mean $( \pm$ SEM) values are indicated by dots and bars. At least 14 mice per group were analyzed in three independent experiments. Statistically significant differences between groups were analyzed by Kruskal-Wallis test and indicated by ${ }^{* *}$ for $p<0.001$.

Additional file 4: Influence of IL-2/anti-IL-2 mAb complex treatment on $T$ cell subsets Mice were infected with 20.000 SFFU of FV and sacrificed at $12 \mathrm{dpi}$. If indicated, Tregs were depleted by repeated injections of DT and mice were stimulated by injections of IL-2/anti-IL-2 mAb complex. Control mice were inoculated with isotype control. Single cell suspensions of splenocytes were stained for characteristic T cell markers ((a) CD4+ T cells; (b) CD8+ T cells and (c) Tregs) as well as activation (CD43) was analyzed using flow cytometry. Tregs were also stimulated with IL-2/anti-IL-2 mAb complex targeting CD25 on cell surface of Tregs (JES6-1) (c). Dotted lines represent the mean activation in naive mice. At least four mice per group were analyzed. Statistically significant differences between FV and FV+IL2/anti-IL-2 (JES6-1) were analyzed by Mann-Whitney test and indicated by* for $\mathrm{p}<0.05$.

\section{Authors' contributions}

ELS designed and performed the experiments, analysed the data, participated in the statistical analysis and wrote the paper. IA, CV and SF performed the experiments. UD conceived and designed the experiments and wrote the paper. KG conceived and designed the experiments, performed the experiments, analysed the data, participated in the statistical analysis and wrote the paper. All authors read and approved the final manuscript.

\section{Acknowledgements}

We thank Adelheid Cerwenka (DKFZ, Heidelberg) for providing us with RMA/S cells. This work was supported by a grant from the University Hospital Essen (IFORES) and by a grant of the Deutsche Forschungsgemeinschaft (TRR 60).

\section{Compliance with ethical guidelines}

\section{Competing interests}

The authors declare that they have no competing interests.

Received: 24 March 2015 Accepted: 16 July 2015

Published online: 30 July 2015

\section{References}

1. Kasaian MT, Leite-Morris KA, Biron CA (1991) The role of CD4+ cells in sustaining lymphocyte proliferation during lymphocytic choriomeningitis virus infection. J Immunol 146:1955-1963

2. Biron CA, Young HA, Kasaian MT (1990) Interleukin 2-induced proliferation of murine natural killer cells in vivo. J Exp Med 171:173-188

3. Willerford DM, Chen J, Ferry JA, Davidson L, Ma A, Alt FW (1995) Interleukin-2 receptor alpha chain regulates the size and content of the peripheral lymphoid compartment. Immunity 3:521-530

4. Setoguchi R, Hori S, Takahashi T, Sakaguchi S (2005) Homeostatic maintenance of natural Foxp3(+) CD25(+) CD4(+) regulatory T cells by interleukin (IL)-2 and induction of autoimmune disease by IL-2 neutralization. J Exp Med 201:723-735
5. Krieg C, Letourneau S, Pantaleo G, Boyman O (2010) Improved IL-2 immunotherapy by selective stimulation of IL-2 receptors on lymphocytes and endothelial cells. Proc Natl Acad Sci USA 107:11906-11911

6. Sakaguchi S, Sakaguchi N, Asano M, Itoh M, Toda M (1995) Immunologic self-tolerance maintained by activated $T$ cells expressing IL-2 receptor alpha-chains (CD25). Breakdown of a single mechanism of self-tolerance causes various autoimmune diseases. J Immunol 155:1151-1164

7. Wildin RS, Ramsdell F, Peake J, Faravelli F, Casanova JL, Buist N et al (2001) $X$-linked neonatal diabetes mellitus, enteropathy and endocrinopathy syndrome is the human equivalent of mouse scurfy. Nat Genet 27:18-20

8. Sakaguchi S, Sakaguchi N, Shimizu J, Yamazaki S, Sakihama T, Itoh M et al (2001) Immunologic tolerance maintained by CD25+ CD4+ regulatory $T$ cells: their common role in controlling autoimmunity, tumor immunity, and transplantation tolerance. Immunol Rev 182:18-32

9. Zelinskyy G, Dietze KK, Husecken YP, Schimmer S, Nair S, Werner T et al (2009) The regulatory T-cell response during acute retroviral infection is locally defined and controls the magnitude and duration of the virusspecific cytotoxic T-cell response. Blood 114:3199-3207

10. Aandahl EM, Michaelsson J, Moretto WJ, Hecht FM, Nixon DF (2004) Human CD4+ CD25+ regulatory $T$ cells control T-cell responses to human immunodeficiency virus and cytomegalovirus antigens. J Virol 78:2454-2459

11. Kinter AL, Horak R, Sion M, Riggin L, McNally J, Lin Y et al (2007) CD25+ regulatory $T$ cells isolated from $\mathrm{HIV}$-infected individuals suppress the cytolytic and nonlytic antiviral activity of HIV-specific CD8+T cells in vitro. AIDS Res Hum Retroviruses 23:438-450

12. Cabrera R, Tu Z, Xu Y, Firpi RJ, Rosen HR, Liu C et al (2004) An immunomodulatory role for $\mathrm{CD} 4(+) \mathrm{CD} 25(+)$ regulatory $T$ lymphocytes in hepatitis C virus infection. Hepatology 40:1062-1071

13. Pedroza-Pacheco I, Madrigal A, Saudemont A (2013) Interaction between natural killer cells and regulatory $T$ cells: perspectives for immunotherapy. Cell Mol Immunol 10:222-229

14. Zimmer J, Andres E, Hentges F (2008) NK cells and Treg cells: a fascinating dance cheek to cheek. Eur J Immunol 38:2942-2945

15. Martin MP, Gao X, Lee J-H, Nelson GW, Detels R, Goedert JJ et al (2002) Epistatic interaction between KIR3DS1 and HLA-B delays the progression to AIDS. Nat Genet 31:429-434

16. Martin MP, Qi Y, Gao X, Yamada E, Martin JN, Pereyra F et al (2007) Innate partnership of HLA-B and KIR3DL1 subtypes against HIV-1. Nat Genet 39:733-740

17. Alter G, Teigen N, Ahern R, Streeck H, Meier A, Rosenberg ES et al (2007) Evolution of innate and adaptive effector cell functions during acute HIV-1 infection. J Infect Dis 195:1452-1460

18. Altfeld M, Goulder P (2007) 'Unleashed' natural killers hinder HIV. Nat Genet 39:708-710

19. Alter G, Heckerman D, Schneidewind A, Fadda L, Kadie CM, Carlson JM et al (2011) HIV-1 adaptation to NK-cell-mediated immune pressure. Nature 476:96-100

20. Matusali G, Tchidjou HK, Pontrelli G, Bernardi S, D'Ettorre G, Vullo V et al (2013) Soluble ligands for the NKG2D receptor are released during HIV-1 infection and impair NKG2D expression and cytotoxicity of NK cells. FASEB J Off Publ Fed Am Soc Exp Biol 27:2440-2450

21. Littwitz E, Francois S, Dittmer U, Gibbert K (2013) Distinct roles of NK cells in viral immunity during different phases of acute Friend retrovirus infection. Retrovirology 10:127

22. Gasteiger G, Hemmers S, Bos PD, Sun JC, Rudensky AY (2013) IL-2-dependent adaptive control of NK cell homeostasis. J Exp Med 210:1179-1187

23. Lindenberg M, Solmaz G, Puttur F, Sparwasser T (2014) Mouse cytomegalovirus infection overrules T regulatory cell suppression on natural killer cells. Virol J 11:145

24. Myers L, Joedicke JJ, Carmody AB, Messer RJ, Kassiotis G, Dudley JP et al (2013) IL-2-independent and TNF-alpha-dependent expansion of Vbeta5+ natural regulatory $T$ cells during retrovirus infection. J Immunol 190:5485-5495

25. Chesebro B, Bloom M, Wehrly K, Nishio J (1979) Persistence of infectious Friend virus in spleens of mice after spontaneous recovery from virusinduced erythroleukemia. J Virol 32:832-837

26. Zelinskyy G, Kraft AR, Schimmer S, Arndt T, Dittmer U (2006) Kinetics of $C D 8+$ effector $T$ cell responses and induced $C D 4+$ regulatory $T$ cell responses during Friend retrovirus infection. Eur J Immunol 36:2658-2670

27. Dietze KK, Zelinskyy G, Liu J, Kretzmer F, Schimmer S, Dittmer U (2013) Combining regulatory $T$ cell depletion and inhibitory receptor blockade 
improves reactivation of exhausted virus-specific CD8+T cells and efficiently reduces chronic retroviral loads. PLoS Pathog 9:e1003798

28. Dietze KK, Zelinskyy G, Gibbert K, Schimmer S, Francois S, Myers L et al (2011) Transient depletion of regulatory T cells in transgenic mice reactivates virus-specific $C D 8+T$ cells and reduces chronic retroviral set points. Proc Natl Acad Sci USA 108:2420-2425

29. Zelinskyy G, Myers L, Dietze KK, Gibbert K, Roggendorf M, Liu J et al (2011) Virus-specific CD8+T cells upregulate programmed death-1 expression during acute friend retrovirus infection but are highly cytotoxic and control virus replication. J Immunol 187:3730-3737

30. Lahl K, Loddenkemper C, Drouin C, Freyer J, Arnason J, Eberl G et al (2007) Selective depletion of Foxp3+ regulatory T cells induces a scurfy-like disease. J Exp Med 204:57-63

31. Huntington ND, Tabarias H, Fairfax K, Brady J, Hayakawa Y, Degli-Esposti MA et al (2007) NK cell maturation and peripheral homeostasis is associated with KLRG1 up-regulation. J Immunol 178:4764-4770

32. Chiossone L, Chaix J, Fuseri N, Roth C, Vivier E, Walzer T (2009) Maturation of mouse NK cells is a 4-stage developmental program. Blood 113:5488-5496

33. Hayakawa Y, Smyth MJ (2006) CD27 dissects mature NK cells into two subsets with distinct responsiveness and migratory capacity. J Immunol 176:1517-1524

34. Ghiringhelli F, Menard C, Terme M, Flament C, Taieb J, Chaput N et al (2005) CD4+ CD25+ regulatory T cells inhibit natural killer cell functions in a transforming growth factor-beta-dependent manner. J Exp Med 202:1075-1085

35. Smyth MJ, Teng MW, Swann J, Kyparissoudis K, Godfrey DI, Hayakawa Y (2006) CD4+ CD25+T regulatory cells suppress NK cell-mediated immunotherapy of cancer. J Immunol 176:1582-1587

36. Manzke N, Akhmetzyanova I, Hasenkrug KJ, Trilling M, Zelinskyy G, Dittmer U (2013) CD4+ T cells develop antiretroviral cytotoxic activity in the absence of regulatory T cells and CD8+ T cells. J Virol 87:6306-6313

37. Boyman O, Sprent J (2012) The role of interleukin-2 during homeostasis and activation of the immune system. Nat Rev Immunol 12:180-190

38. Boyman O, Kovar M, Rubinstein MP, Surh CD, Sprent J (2006) Selective stimulation of T cell subsets with antibody-cytokine immune complexes. Science 311:1924-1927

39. Belkaid Y, Rouse BT (2005) Natural regulatory T cells in infectious disease. Nat Immunol 6:353-360

40. Duppach J, Francois S, Joedicke JJ, Dittmer U, Kraft AR (2014) Expanded regulatory $T$ cells in chronically Friend retrovirus infected mice suppress immunity to a mCMV superinfection. J Virol 88(23):13892-13896

41. Lafaille FG, Pessach IM, Zhang SY, Ciancanelli MJ, Herman M, Abhyankar A et al (2012) Impaired intrinsic immunity to HSV-1 in human iPSC-derived TLR3-deficient CNS cells. Nature 491:769-773
42. Cao X, Cai SF, Fehniger TA, Song J, Collins LI, Piwnica-Worms DR et al (2007) Granzyme B and perforin are important for regulatory $T$ cellmediated suppression of tumor clearance. Immunity 27:635-646

43. Joedicke JJ, Dietze KK, Zelinskyy G, Dittmer U (2014) The phenotype and activation status of regulatory $T$ cells during Friend retrovirus infection. Virologica Sinica 29:48-60

44. Sitrin J, Ring A, Garcia KC, Benoist C, Mathis D (2013) Regulatory T cells control NK cells in an insulitic lesion by depriving them of IL-2. J Exp Med 210:1153-1165

45. Gasteiger G, Hemmers S, Firth MA, Le Floc'h A, Huse M, Sun JC et al (2013) IL-2-dependent tuning of NK cell sensitivity for target cells is controlled by regulatory T cells. J Exp Med 210:1167-1178

46. Lee S, Margolin K (2011) Cytokines in cancer immunotherapy. Cancers 3:3856-3893

47. Shevach EM (2012) Application of IL-2 therapy to target T regulatory cell function. Trends Immunol 33:626-632

48. Newman RG, Dee MJ, Malek TR, Podack ER, Levy RB (2014) Heat shock protein vaccination and directed IL-2 therapy amplify tumor immunity rapidly following bone marrow transplantation in mice. Blood 123:3045-3055

49. Kamimura D, Sawa Y, Sato M, Agung E, Hirano T, Murakami M (2006) IL-2 in vivo activities and antitumor efficacy enhanced by an anti-IL-2 mAb. J Immunol 177:306-314

50. Hamilton SE, Schenkel JM, Akue AD, Jameson SC (2010) IL-2 complex treatment can protect naive mice from bacterial and viral infection. J Immunol 185:6584-6590

51. Levin AM, Bates DL, Ring AM, Krieg C, Lin JT, Su L et al (2012) Exploiting a natural conformational switch to engineer an interleukin-2 'superkine'. Nature 484:529-533

52. Margolin K, Atkins MB, Dutcher JP, Ernstoff MS, Smith JW 2nd, Clark Jl et al (2007) Phase I trial of BAY 50-4798, an interleukin-2-specific agonist in advanced melanoma and renal cancer. Clin Cancer Res Off J Am Assoc Cancer Res 13:3312-3319

53. Dittmer U, Brooks DM, Hasenkrug KJ (1998) Characterization of a liveattenuated retroviral vaccine demonstrates protection via immune mechanisms. J Virol 72:6554-6558

\section{Submit your next manuscript to BioMed Central and take full advantage of:}

- Convenient online submission

- Thorough peer review

- No space constraints or color figure charges

- Immediate publication on acceptance

- Inclusion in PubMed, CAS, Scopus and Google Scholar

- Research which is freely available for redistribution

Submit your manuscript at 